\title{
Calcium distribution during anther development in Impatiens balsamina
}

\author{
S.J. YANG*, W.Y. LIANG, J. SHI, L. PENG, and R. ZHENG \\ School of Life Sciences, Ningxia University, Yinchuan 750021, Ningxia, P.R. China
}

\begin{abstract}
The subcellular localization of loosely bound calcium in developing anthers of Impatiens balsamina (rose balzam) was investigated using potassium antimonate to precipitate the ions. At the sporogenous stage, few calcium precipitates were found in the young anthers. During meiosis of the microspore mother cell, the amount of calcium precipitates increased both inside these cells and in their cell walls. After meiosis, the resulting microspores accumulated abundant calcium precipitates in their cytoplasm and vacuoles. The microspores underwent mitosis to produce a bicellular pollen grain, in which the large vacuoles disintegrated, and the calcium precipitates of the vegetative cell were again localized in the cytoplasm. The amount of precipitates decreased during pollen maturity and finally disappeared from the inside of the pollen grains and accumulated on the pollen walls.
\end{abstract}

Additional key words: loosely bound calcium, microspore mother cell, pollen grain.

\section{Introduction}

Calcium is an important ion for all organisms as it is involved in the processes of growth and development (Bush 1995). In plant cells, calcium can be divided into free calcium, bound calcium, and stored calcium. Free calcium exists in cells in a low amount, less than $10^{-6} \mathrm{M}$, which is difficult to detect in tissues. It is the main component of intracellular calcium signal, and its instantaneous increase will lead to physiological and biochemical changes in cells. The bound calcium often binds closely with other structural components in cells, in the form of calcium oxalate, calcium phosphate, and calcium carbonate, and is not easy to release $\mathrm{Ca}^{2+}$. Storage for most of the total calcium often exits in the organelles and the cell wall. When the cell physiological activities need to increase the $\mathrm{Ca}^{2+}$ content, the calcium is released from storage sites and transported to other parts of the cells. This kind of calcium is also known as loosely bound calcium. The spatial distributions of calcium binding sites can be identified with the method of antimonate precipitation. The distribution of $\mathrm{Ca}^{2+}$ in the cell is very uneven, and the content of $\mathrm{Ca}^{2+}$ in the intercellular space, cell walls, and vacuoles is much higher than that in the cytoplasm. Therefore, it is often used to refer to the intercellular space and cell wall as extracellular calcium pool, while vacuoles and other organelles as intracellular calcium pool (Denninger et al. 2014). The universality of calcium as an intracellular messenger depends on the dynamics of its spatial and temporal release from calcium stores. Intracellular calcium regulates a wide variety of cellular processes. Calcium signals are generated by both the release of stored calcium from the endoplasmic reticulum (ER) and the influx of extracellular calcium across the plasma membrane.

In flowering plants, calcium plays an essential signalling, physiological, and regulatory role during sexual reproduction. Elevation of calcium amounts in the cells is an accurate predictor of plant fertility $(\mathrm{Ge}$ et al.2007). Among examinations of calcium involvement in reproduction, calcium effects on pollen germination and pollen tube elongation have long been studied based on in vitro experiments (Feijó et al.1995, Malho and Trewavas 1996, Pierson et al. 1996). The spatial-temporal distribution characteristics of calcium in the ovaries and ovules of some flowering plants have been examined in recent years, and the highest calcium content occurs in synergids (Chaubal and Reger 1992, Tian and Russell 1997, Tian et al. 2000). The distribution of large amounts of calcium ions in the micropyle and synergids may have the effect of attracting pollen tubes to the embryo sac. Recent studies showed that once a pollen tube arrived to the synergid, $\mathrm{Ca}^{2+}$ oscillations spread towards the chalazal pole and $\mathrm{Ca}^{2+}$ content in the synergids peaked upon pollen tube rupture (Iwano et al. 2012, Denninger et al. 2014, Hamamura et al. 2014). These characteristic changes in $\mathrm{Ca}^{2+}$ distribution in the synergids are closely associated with the entry of the pollen tube into the female gametophyte. The spatial-temporal features of $\mathrm{Ca}^{2+}$ distribution during anther development are poorly understood, and the role of $\mathrm{Ca}^{2+}$ in this process is still unclear.

Rose balsam (Impatiens balsamina) is an ornamental

Submitted 19 February 2019, last revision 20 October 2019, accepted 29 November 2019.

Abbreviations: MMC - microspore mother cell; SC - sporogenous cell; gc - generative cell.

*Corresponding authors; e-mail: nxyang11@126.com 
plant used also in traditional Chinese medicine (Kang et al. 2013, Oku and Ishiguro 2002). Many active ingredients have been isolated from I. balsamina, including phenolics, flavonols, anthocyanin pigments, and saponins (Sakunphueak et al.2013, Shin et al. 2015). Here, we report the distribution of calcium during anther developing in I. balsamina with the aim to find the relationship between $\mathrm{Ca}^{2+}$ distribution and anther development. Moreover, this study may serve as a theoretical basis for the sexual reproduction of I. balsamina.

\section{Materials and methods}

Impatiens balsamina L. was grown on the campus field of Ningxia University, Yinchuan, China. The anthers were collected and examined by using a microscope to determine their different developmental stages according to the pollen structural features.

In present study, we use potassium antimonate to precipitate the loosely bound calcium which has a low affinity for mobile anions. The principle is that calcium antimonite precipitates cannot be penetrated by electrons and enable their visualization as dark spots under the electron microscope (Wick and Hepler 1982, Tian and Russell 1997, Ge et al. 2007). Potassium pyroantimonate precipitation has been used to locate calcium in rice (Tian et al. 1998), wheat (Meng et al. 2000) and other plants. The anthers were cut in half and fixed at $4{ }^{\circ} \mathrm{C}$ for $5 \mathrm{~h}$ in $2.5 \%$ glutaraldehyde (v/v) in $0.1 \mathrm{M} \mathrm{KH}_{2} \mathrm{PO}_{4}$ buffer $(\mathrm{pH} 7.8)$ which contains $1 \%(\mathrm{~m} / \mathrm{v})$ potassium antimonite $\left(\mathrm{K}_{2} \mathrm{H}_{2} \mathrm{Sb}_{2} \mathrm{O}_{7} \cdot 4 \mathrm{H}_{2} \mathrm{O}\right)$, followed by washing three times for $30 \mathrm{~min}$ in the $0.1 \mathrm{M} \mathrm{KH}_{2} \mathrm{PO}_{4}$ buffer (pH 7.5) containing $1 \%$ potassium antimonite. Then the samples were postfixed in $1 \%(\mathrm{~m} / \mathrm{v})$ buffered osmium tetroxide $\left(\mathrm{OsO}_{4}\right)$ containing $1 \%$ potassium antimonate for $16 \mathrm{~h}$ and maintained at $4{ }^{\circ} \mathrm{C}$. The samples were then washed three times for $30 \mathrm{~min}$ in each buffer. After dehydrating in a graded acetone series from 10 to $100 \%$, the samples (at least 10 anthers of each stage) were embedded in Epon 812 resin. The samples were sliced at $80 \mathrm{~nm}$ thick with an ultramicrotome, and stained with $2 \%(\mathrm{~m} / \mathrm{v})$ uranyl acetate for $20 \mathrm{~min}$ and observed with a JEM-2100 (JEOL, Tokyo, Japan) transmission electron microscope.

\section{Results}

Calcium distribution was studied from the sporogenous cell to mature pollen grain stages using transmission electron and light microscopy to determine whether the calcium affect the pollen development in I. balsamina or not. When the anthers of I. balsamina were cut transversely, the sections had a butterfly shape comprising four microsporangia. In the transverse view, the anther wall consisted of five different cell layers; one layer of epidermis and endothecium, two middle layers, and one layer of tapetum. The locules contain the microspore mother cells (MMCs) or pollen grains (Fig. 1A). By observing the morphological and structural features of the pollen grains, their development can be artificially divided into the following stages: sporogenous, MMC, tetrad, microspore, early two-celled pollen, and mature pollen.

In anthers at the sporogenous cell stage, the anther wall had formed five different layers. The cells contained many small organelles, especially plastids and scattered fragments of the endoplasmic reticulum. Some calcium precipitation was observed in the sporogenous cells (Fig. 1B); however, these precipitates also accumulated at the radial and inner tangential walls of the tapetal cells, but not in their cytoplasm (Fig. 1C). The organelles observed within the tapetal cells were mainly mitochondria, scattered fragments of endoplasmic reticulum, and a Golgi apparatus.

During anther development, the sporogenous cells transitioned into MMCs, which involved two clear changes; 1) the cellular arrangement of MMCs is looser than that of the sporogenous cells, so that there are more intercellular spaces, and 2) MMCs begin to accumulate callose in walls. The thick callose wall was apparent characteristics to distinguish from the sporogenous cells (Fig. 1D). The MMCs also produced more plastids with low electron densities and contained small starch grains. We observed more calcium precipitates in the MMCs, most of which were accumulated in the mitochondria (Fig. 1E). After the separation of the two nuclei during MMC meiosis, the starch-containing plastids mostly accumulated at the equatorial line between the two nuclei, while the calcium precipitates were dispersed throughout the cytoplasm (Fig. 1F). Concurrently, the amount of precipitates at the callose wall of the MMCs declined sharply. At this stage, there was a layer of the precipitates on the inner tangential walls of the tapetum 1 cells, and large vacuoles were also observed in a polar centripetal orientation in these cells (Fig. 2A). The calcium precipitates in the tapetal cells mainly accumulated in mitochondria that had lost their inner membrane (cristae) but retained their outer membrane to become a small vacuole (Fig. 2B). The structural features of the tapetal cells suggest that they are involved in the active transport of $\mathrm{Ca}^{2+}$ from the anther wall to the locule.

The first meiotic division of the MMCs was not followed by cytokinesis in I. balsamina, instead a binucleate cell was formed (Fig. 1E). The two nuclei synchronously underwent the second meiotic division, forming a tetranucleate cell which then completed cytokinesis, with callose walls forming centripetally between the four nuclei (Fig. 2C). The callose walls meet in the center of the tetranucleate cell, dividing the four nuclei into four microspores in a tetrahedral tetrad (Fig. 2D). The starch granules present in the center of the MMCs were translocated to a dispersed position in the four microspores. During meiosis, the calcium precipitates in the MMCs were observed to decrease; however, there are still calcium precipitates detected mainly in some small vacuoles of the tapetal cells (Fig. 2E).

Following the dissolution of the callose wall, the four microspores were released from the tetrad, and there is a large centrally nucleus in the early microspores (Fig. 2F). The early microspores already possessed 
YANG et al.
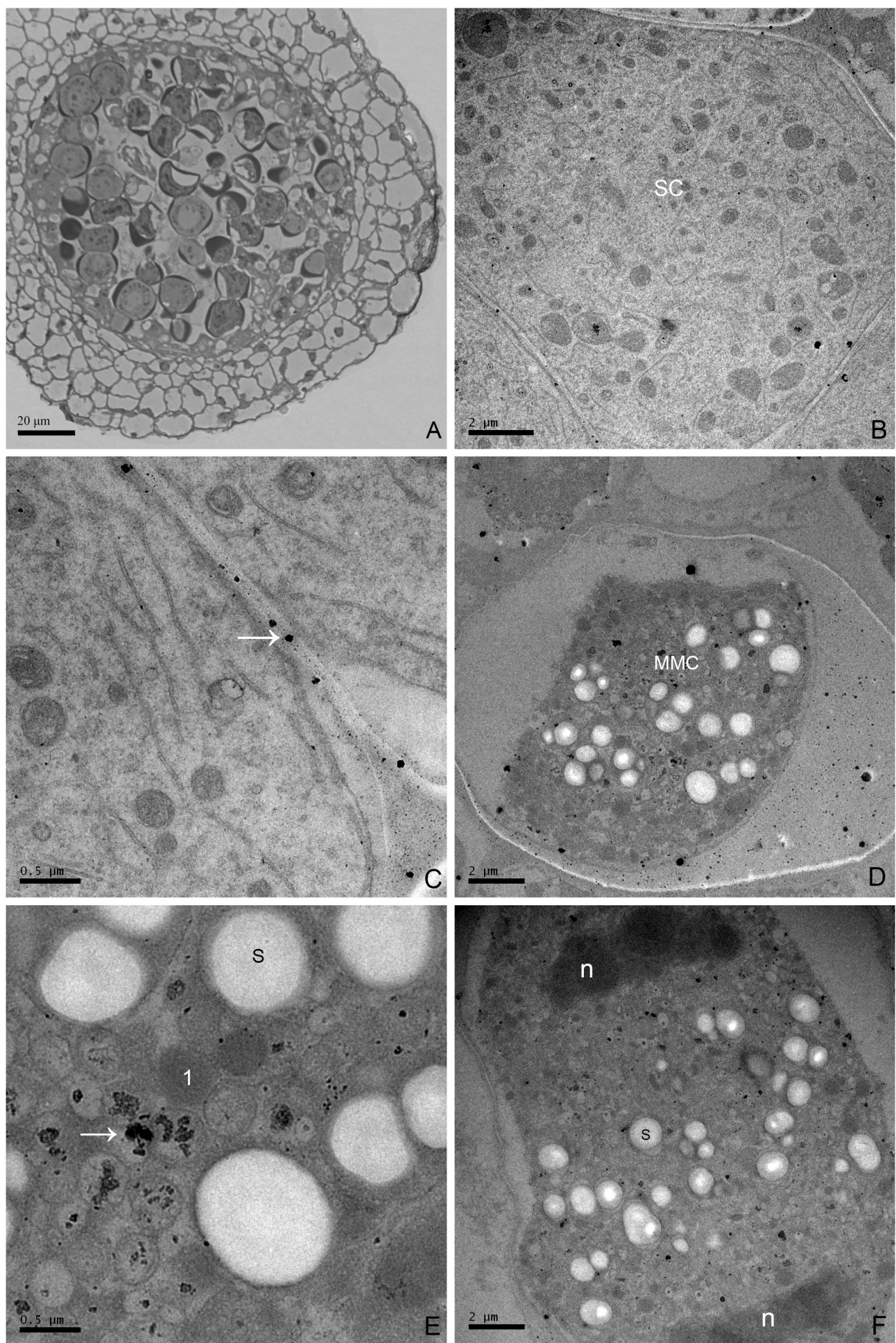

Fig. 1. Calcium distribution in the anthers of Impatiens balsamina at the sporogenous stage and the stage of microspore mother cell. $A$ - A locule of an anther of I. balsamina at a low magnification to show anther structure. Magnification is 200; the scale bar is $40 \mu \mathrm{m}$. $B$ No calcium precipitates were present in sporogenous cells (SC). $C$ - Some calcium precipitates were present in the wall between tapetal cells (the arrow). D - Many starch bodies were present in the microspore mother cell (MMC). The majority of calcium precipitates were located in the callose cell wall, but some calcium precipitates were present in the cytoplasm. $E$ - Precipitates in the MMCs were accumulated in small vacuoles (the arrow). $F$ - During the first division of MMC, organelles, especially plastids and starch bodies, were located at the equatorial line between the two nuclei (n); however, the calcium precipitates were dispersed throughout the cytoplasm surrounding the two nuclei. 

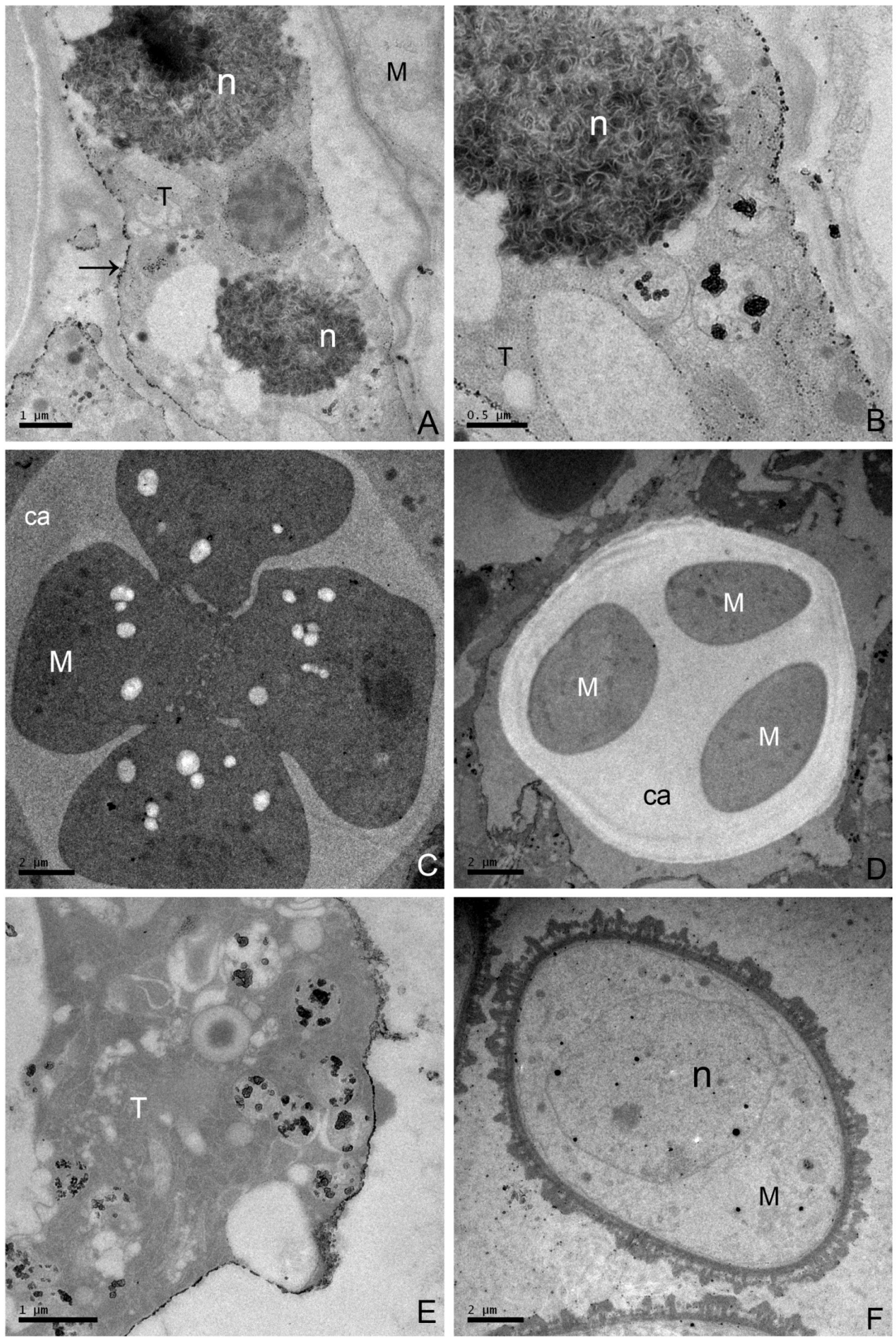

Fig. 2. Calcium distribution in the anthers of Impatiens balsamina at the stage of microspore. $A$ - During microspore mother cell (MMC) meiosis, the calcium precipitates in the tapetal cells became more abundant and occupied a polar distribution on their inner tangential walls (the arrow); $\mathrm{n}$ - nucleus, $\mathrm{M}$ - microspore, $\mathrm{T}$ - tapetum. $B$ - In the tapetal cells, the precipitates were mainly located in mitochondria. $C$ - During the cytokinesis of the MMCs, the callose walls grew centripetally to form furrows, which met in the center of the cell and simultaneously divided the four nuclei into four microspores; ca - callose. $D$ - A tetrahedral tetrad of microspores containing no calcium precipitates. $E$ - At this stage, the tapetal cell and its inner tangential wall still contained many precipitates. $F$ - Early microspores, which still hade a centrally located nucleus, contained some calcium precipitates but none in the cytoplasm. 
YANG et al.
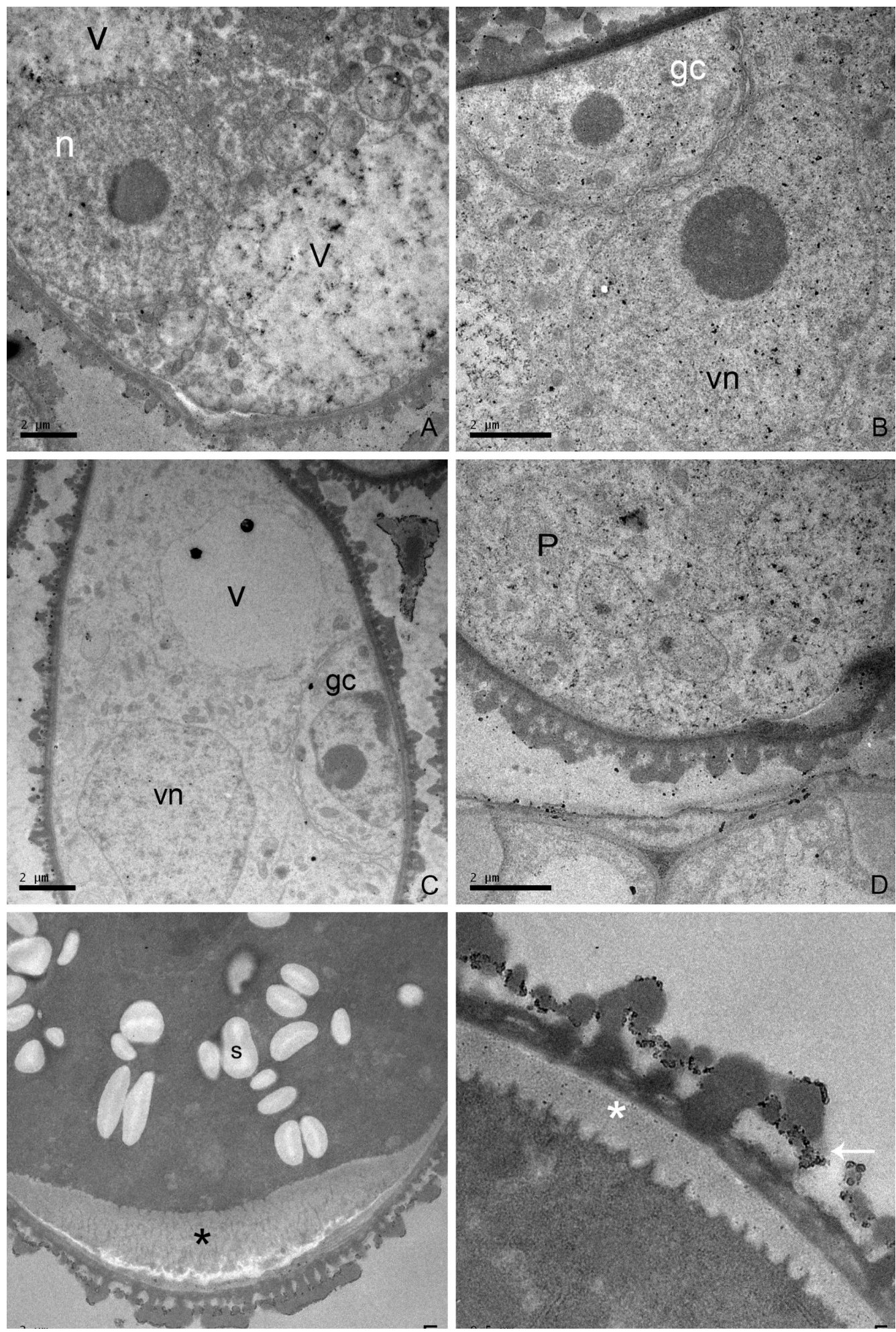

Fig. 3. Calcium distribution in the anthers of Impatiens balsamina at the stage of bicellular pollen. $A$ - The developing microspore formed large vacuoles $(\mathrm{V})$ that pushed the nucleus (n) to the side of the cell. The vacuoles contained many precipitates. $B$ - After division of microspore, the vegetative nucleus (vn) was in a close proximity to the generative cell ( $\mathrm{gc}$ ). Both contained some small precipitates. $C$ - Large calcium precipitates appeared in the large vacuoles (V) of the bicellular pollen, but not in its cytoplasm. $D$ - When large vacuoles decomposed, the precipitates accumulated in the cytoplasm of the bicellular pollen (P). $E$ - At anthesis, the dense cytoplasm of the mature pollen contained many starch bodies (s) but no calcium precipitates. The intine of the pollen was thickened (the asterisk). $F$ - Many precipitates accumulated in the tectum (the arrow) of the pollen wall. The wall ingrowths of intine were very obvious (the asterisk). 
a pollen exine consisting of three layers, which is a typical feature of I. balsamina pollen. There were more calcium precipitates in the early microspore in comparison with the tetrad cells, and they were initially located in the nucleus. During development, several large vacuoles appeared in the microspores, whose nuclei were translocated to the edge of the cell. During this process, the abundance of the calcium precipitates increased overall; they were most abundant in the vacuoles, but their amount decreased in the nuclei (Fig 3A). During microspore development, the tapetal cells degenerated and disappeared.

I. balsamina pollen grains have a rugby-ball-like morphology; their diameter is about half of the length of the polar axis. During pollen development, the nucleus of the microspore moved to the equatorial line, where it underwent an asymmetrical mitotic division to form a bicellular pollen grain consisting of a larger vegetative cell and a smaller generative cell. Initially, the nucleus of the vegetative cell is in close proximity to the generative cell (Fig. $3 B$ ); however, the vegetative nucleus then moves away from the generative cell (Fig. $3 C$ ). At the stage of bicellular pollen, the large vacuoles in the vegetative cell decomposed into several small vacuoles containing some calcium precipitates. Following the disappearance of the pollen vacuoles, the calcium precipitates appeared again in the cytoplasm (Fig. $3 D$ ), indicating that $\mathrm{Ca}^{2+}$ contained in the vacuoles moves back into the cytoplasm.

In mature pollen, polysaccharide metabolism increases, and many starch grains were therefore observed (Fig. $3 E$ ). No calcium precipitates were presented in the cytoplasm of the mature pollen; instead, they accumulated at the pollen wall (Fig. $3 F$ ).

\section{Discussion}

Microsporocyte differentiation concerning number and ultrastructure of organelles facilitates the transition of microsporocytes from the sporophytic phase to the gametophytic phase. The MMCs undergo meiosis and subsequent cytokinesis, forming isobilateral tetrads. Following the dissolution of the callose wall and release of free and vacuolated microspores, mitotic divisions lead to the formation of mature, two-celled pollen grains in I. balsamina. Many studies about anther and pollen development have been done in Arabidopsis that is used in a lot of research (Wilson and Zhang 2009), and the process of development is a complex and important process that is associated with the release of viable pollen and plant fertilization (Gomez et al. 2015). Anther development involves significant morphological and physiological changes, such as the formation of the callose wall in the MMCs and the later degradation of callose wall in the tetrads, the unequal division of microspores, the degeneration of the tapetal cells, the formation of the large vacuole and the high polarity of the microspore cytoplasm, the formation of the sporopollenin wall, and the formation of fibrous bands in the endothecial cells (Bhojwani and Bhatnagar 1974). All of these changes are programmed steps in anther development, and the disruption of any of them will affect pollen fertility. However, the mechanisms regulating individual steps in anther development remain unclear.

Depending on its cellular location, $\mathrm{Ca}^{2+}$ regulates many cellular functions including coordination of the activities of certain enzymes. Calcium acts as signalling ion between cells, serving as charge generators, forming an essential component of the cell structure, and possibly acting as a secondary messenger (Bush 1995). There have been several investigations concerning male sterility (Tian et al. 1998, Meng et al. 2000) and the distribution of calcium precipitates in fertile anther are significantly different from those of sterile anthers, suggesting that $\mathrm{Ca}^{2+}$ is associate with regulating the development of viable pollen. Several studies have demonstrated that $\mathrm{Ca}^{2+}$ accumulates in pollen in a particular spatial-temporal distribution during anther development (Qiu et al.2009, Weietal.2015, Li et al. 2017). In young lettuce anthers after MMC meiosis, a significant increase in the amount of calcium precipitate is found not only in the nucleus and cytoplasm, but also in exine of early microspores, suggesting that regulation of $\mathrm{Ca}^{2+}$ dynamics may be directly linked with anther development (Wei et al. 2015). The formation of a large vacuole was related to the decrease of calcium precipitates during later microspore development stage (Qiu et al. 2009). In Camellia oleifera, abundant calcium precipitates are observed in the callose wall of the MMCs before meiosis, then appear in the small vacuoles of the early microspores. In the late microspores with large vacuoles, the precipitates decreased obviously, indicating that $\mathrm{Ca}^{2+}$ dissolved into the large vacuole (Wei et al. 2015). Recently Li et al. (2017) confirmed that, during wheat microspore development, calcium precipitates first appear in the mitochondria in which the cristae of the inner membrane are destroyed, but the outer membrane remain intact. These findings suggest that the small vacuoles may originate from mitochondria under the influence of $\mathrm{Ca}^{2+}(\mathrm{Li}$ et al. 2017). In the present study, two increases in calcium precipitate abundance were observed during microspore development. Firstly, the particles accumulated in the MMC and its callose wall (Fig. 1D,E), which might be related to the formation of the callose wall. Secondly, the presence of numerous calcium particles in vacuoles of the microspores was observed (Fig. $3 A$ ), similarly as in the anthers of Camellia oleifera (Wei et al. 2015) and wheat (Li et al. 2017). Increases in $\mathrm{Ca}^{2+}$ during pollen development may be involved in the formation of vacuoles.

\section{References}

Bhojwani, S.S., Bhatnagar, S.P.: The Embryology of Angiosperms. - Vikas Publishing House, New Delhi - Bombay - Bangalore - Calcutta - Kanpur 1974.

Bush, D.: Calcium regulation in plant cells and its role in signaling. - Annu. Rev Plant Physiol. 46: 95-122, 1995.

Chaubal, R.J., Reger, B.: Calcium in the synergid cells and other regions of pearl millet ovaries. - Sex Plant Reprod. 5: 34-46, 1992.

Denninger, P., Bleckmann, A., Lausser, A., Vogler, F., Ott, T., Ehrhardt, D.W., Frommer, W.B., Sprunck, S., Dresselhaus, T., Grossmann, G.: Male-female communication triggers 
calcium signatures during fertilization in Arabidopsis. - Nat Commun. 5: 4645, 2014.

Feijó, J., Malhó, R., Obermeyer, G.: Ion dynamics and its possible role during in vitro pollen germination and tube growth. - Protoplasma 187: 155-167, 1995.

Ge, L.L., Tian, H.Q., Russell, S.D.: Calcium function and distribution during fertilization in angiosperms. - Amer. J. Bot. 94: 1046-1060, 2007.

Gomez, J.F., Talle, B., Wilson, Z.A.: Anther and pollen development: a conserved developmental pathway. - J. integr. Plant Biol. 57: 876-891, 2015.

Hamamura, Y., Nishimaki, M., Takeuchi, H., Geitmann, A., Kurihara, D., Higashiyama, T.: Live imaging of calcium spikes during double fertilization in Arabidopsis. - Nat. Commun. 5: 4722, 2014.

Iwano, M., Ngo, Q.A., Entani, T., Shiba, H., Nagai, T., Miyawaki, A., Isogai, A., Grossniklaus, U., Takayama, S.: Cytoplasmic $\mathrm{Ca}^{2+}$ changes dynamically during the interaction of the pollen tube with synergid cells. - Development 139: 4202-4209, 2012.

Kang, S.N., Goo, Y.M., Yang, M.R., Ibrahim, R.I., Cho, J.H., Kim, I.S., Lee, O.H.: Antioxidant and antimicrobial activities of ethanol extract from the stem and leaf of Impatiens balsamina L. (Balsaminaceae) at different harvest times. Molecules 18: 6356-6365, 2013.

Li, D.X., Hu, H.Y., Li, G., Ru, Z.G., Tian, H.Q.: Calcium controls the formation of vacuoles from mitochondria to regulate microspore development in wheat. - Plant Reprod. 30: 131139, 2017.

Malho, R., Trewavas, A.J.: Localized apical increases of cytosolic free calcium control pollen tube orientation. - Plant Cell 8: 1935-1949, 1996.

Meng, X.H., Wang, J.B., Li, R.Q.: Effect of photoperiod on calcium distribution in photoperiod-sensitive cytoplasmic male-sterile wheat during anther development. - Acta bot. sin. 42: $15-22,2000$
Oku, H., Ishiguro, K.: Cyclooxygenase-2 inhibitory 1,4-naphthoquinones from Impatiens balsamina L. - Biol. Pharm. Bull. 25: 658-660, 2002.

Pierson, E.S., Miller, D.D., Callaham, D.A., Van Aken, J., Hackett, G., Hepler, P.K.: Tip-localized calcium entry fluctuates during pollen tube growth. - Dev Biol. 174: 160-173, 1996.

Qiu, Y.L., Liu, R.S., Wei, D.M., Tian, H.Q.: Calcium distribution in developing anthers of lettuce. - Ann. Bot. fennici 46: 101106, 2009.

Sakunphueak, A., Tansakul, P., Umehara, K., Noguchi, H., Panichayupakaranant, P.: Effect of methionine on production of naphthoquinones in Impatiens balsamina root cultures and detection of some secondary metabolites. - Pharm. Biol. 51: 36-41, 2013.

Shin, J.A., Ryu, M.H., Kwon, K.H., Choi, B., Cho, S.D.: Downregulation of Akt by methanol extracts of Impatiens balsamina L. promotes apoptosis in human oral squamous cell carcinoma cell lines. - J. Oral Pathol. Med. 44: 420-428, 2015.

Tian, H.Q., Kuang, A.X., E. Musgrave, M., Russell, S.D.: Calcium distribution in fertile and sterile anthers of a photoperiodsensitive genic male-sterile rice. - Planta 204: 183-192, 1998.

Tian, H.Q., Russell, S.D.: Calcium distribution in fertilized and unfertilized ovules and embryo sacs of Nicotiana tabacum L. - Planta 202: 93-105, 1997.

Tian, H.Q., Zhu, H., Russell, S.D.: Calcium changes in ovules and embryo sacs of Plumbago zeylanica L. - Sex Plant Reprod. 13: 11-20, 2000.

Wei, D.M., Gao, C., Yuan, D.Y.: Calcium distribution during anther development in oil tea (Camellia oleifera Abel.). - J. amer. Soc. hort. Sci. 140: 88-93, 2015.

Wick, S.M., Hepler, P.K.: Selective localization of intracellular $\mathrm{Ca}^{2+}$ with potassium antimonate. - J. Histochem Cytochem. 30: 1190-1204, 1982.

Wilson, Z.A., Zhang, D.B.: From Arabidopsis to rice: pathways in pollen development. - J. exp. Bot. 60: 1479-1492, 2009. 